\title{
Analysis of hybrid PMN/Terfenol broadband transducers in mechanical series configuration
}

\author{
Patrick R. Downey ${ }^{1}$, Marcelo J. Dapino ${ }^{2 *}$, and Ralph C. Smith ${ }^{3}$ \\ ${ }^{1,2}$ Mechanical Engineering Department, The Ohio State University, Columbus, OH 43210 \\ ${ }^{3}$ Center for Research in Scientific Computation, North Carolina State University, Raleigh, NC 27695
}

\begin{abstract}
This paper presents recent advances in the design and characterization of hybrid transducers incorporating magnetostrictive and electrostrictive elements. In order to analyze and validate the properties inherent to hybrid concepts, a transducer was designed and constructed through a mechanical series arrangement of a PMN-PT stack and a Terfenol-D rod. This configuration provides a double resonant frequency response that can be tuned for a variety of applications. The primary objective of this study lies in the determination of the design criteria for achieving maximum transducer bandwidth on the $1-6 \mathrm{kHz}$ range. To this end, a linear system model was developed utilizing concepts from vibrations, electroacoustic theory, and linearized constitutive relationships for each class of smart material for low to moderate drive levels. This model provides a means of completely describing the system response and the interactions between electrical and mechanical domains for this hybrid transducer. Experimental data collected from the test device indicate that the measured modes of vibration and resonance peaks agree with the theoretical results, and that the desired bandwidth has been achieved.
\end{abstract}

\section{INTRODUCTION}

Hybrid smart structures containing multiple active materials can exhibit unique attributes and advantages over single element ones. Each material can have different mechanical, electrical, and thermal regimes, as well as unique coupling interactions among regimes. The complementary properties of certain classes of active materials can be employed effectively to design hybrid systems which augment the performance characteristics of or add functionality to any one individual material. Specific metrics that have been targeted for improvement by means of hybrid designs primarily include energy efficiency ${ }^{1}$ and frequency bandwidth ${ }^{2}$. Energy efficiency gains stem from the fact that more mechanical output can be achieved with less electrical energy if a system's available electrical energy is shared between different active elements. In practice, these gains have not been fully realized in hybrid designs. The focus of this investigation is the development of nonlinear models for broadband transducers which employ the complementary electrical and mechanical properties of magnetostrictive and electrostrictive materials. By addressing hysteresis effects and the multiregime coupling both at the transducer level and between the constituent active elements, nonlinear models facilitate design optimization and model-based control with a degree of efficacy which linear models do not. In addition, such models could provide new design criteria for the implementation of the high energy efficiencies thought to be achievable by hybrid architectures.

Conventional Tonpilz sonar transducers employ a single smart material element, usually a piezoelectric stack, but recent U.S. Navy research has been aimed at enhancing the frequency bandwidth of high power Tonpilz transducers through hybrid electrostrictive/magnetostrictive architectures. It has been demonstrated that such architectures can produce increased velocity output in the low frequency range when compared with conventional devices. One design generates over $1 \mathrm{kHz}$ of extra bandwidth on the low frequency range through a mechanical series arrangement of a magnetostrictive section and a piezoelectric section ${ }^{2}$. Key properties that facilitate this operation are an inherent 90degree phase shift between the velocities of the two sections and a natural difference in the speed of sound in the two materials. In addition, the complementary electrical properties of each section results in transducer electrical self tuning under certain drive conditions. Because of the constitutive nonlinearities of the active elements and the coupling among electrical, magnetic, elastic, and thermal regimes, the efficient design and control of hybrid transducers necessitates nonlinear models for the strain, stress and polarization as a function of external inputs. To this end, this paper presents

* Corresponding Author - Email: dapino.1@osu.edu, Telephone: (614) - 688 - 3689 
ongoing research in the modeling of Tonpilz hybrid architectures, which has the ultimate goal of developing optimization tools through nonlinear modeling. Although the model presented in the paper has been linearized, it is emphasized that the methodology and model construction can be naturally extended to employ existing nonlinear techniques ${ }^{3,4,5,6,7}$. The extension of the model to nonlinear regimes is currently under investigation.

The transducer designed here has the Tonpilz configuration, with two smart material sections combined in mechanical series forming a double resonant system in which the lower resonance is controlled by a Terfenol-D element and the higher resonance by a lead magnesium niobate - lead titanate (PMN-PT) stack. Each material exhibits quasi-linear displacements when subjected to low-signal magnetic or electric fields, respectively. The Terfenol-D is driven by a wound wire solenoid that generates a magnetic field, and it electrically represents an inductor in series with a small resistance. A magnetic return path is built around the drive coil and rod to ensure consistent flux through the rod. The PMN-PT stack is driven by application of direct voltage, and electrically it provides most of the capacitance in the system. The two sections are connected in electrical parallel, and thus share energy near resonance.

A system model describing the dynamic behavior of the transducer has been developed in three steps. In the first, a linear vibrations framework is used to model the mechanical regime. The second model component utilizes electroacoustics theory to describe the coupling between the mechanical and electrical regimes and to identify several physical system parameters. The third step involves the development of relations between stress, strain, and applied field, as well as the characterization of material properties. The resulting set of linearized equations is used to quantify the dependence of material properties with drive and acoustic loading conditions, as well as the transducer frequency response functions velocity per voltage and voltage per current.

\section{MECHANICAL MODEL}

The transducer architecture employed here consists of a mechanical series arrangement of electrostrictive and magnetostrictive sections joined through a rigid center mass, with oscillating head and tail masses at opposite ends. This configuration provides a double resonant frequency response which is used to facilitate broad frequency bandwidth operation. For modeling purposes, each smart material section behaves mechanically as a tunable compliance arranged in parallel with a damper as described by the three-degree-of-freedom linear vibratory system shown in Figure 1.

This mechanical model is analyzed to determine the ideal mass and stiffness ratios to achieve the largest bandwidth possible over the $1-6 \mathrm{kHz}$ frequency range. Consistent with prior work, it was determined that optimum bandwidth is achieved when using a head : center : tail mass ratio of approximately $1: 2: 2.5^{2}$. Two natural modes of vibration exist that dominate the motion of the system, the third being a rigid translational mode. Recognizing that the Terfenol-D rod is less stiff than the electrostrictive stack for the dimensions employed, the lower resonance is controlled by the Terfenol-D section, and is characterized by the head and center masses lumped together vibrating out of phase with respect to the tail mass. The PMN-PT stack controls the upper resonance, where the tail mass essentially decouples from the system and the head and center masses are out of phase with each other. These motions are described by the equations derived from Figure 1 (b) for the PMN-PT high frequency circuit,

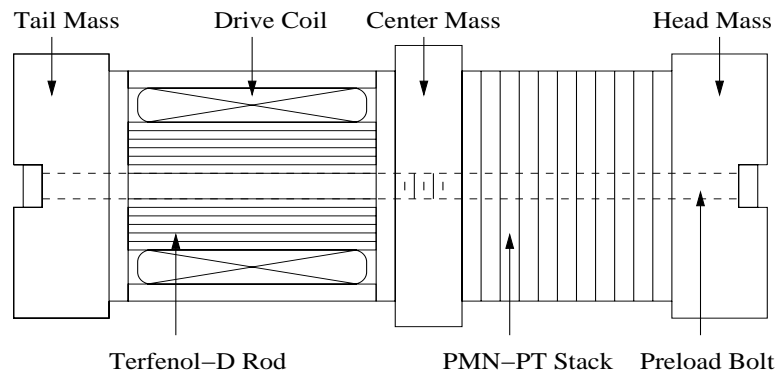

(a)

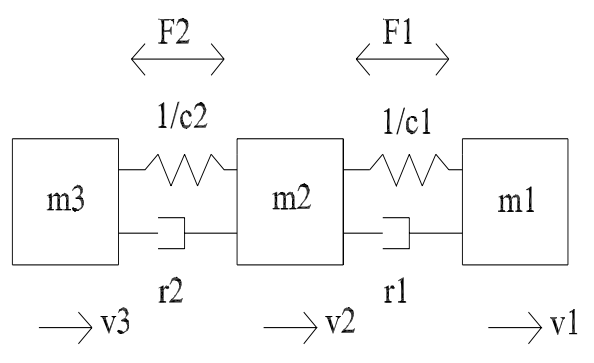

(b)

Figure 1: Diagram of (a) the general transducer design, and (b) the corresponding vibratory model. 


$$
\begin{gathered}
m_{1} s v_{1}+r_{1} v_{1}+v_{1} /\left(s c_{1}\right)=F_{1}+r_{1} v_{2}+v_{2} /\left(s c_{1}\right) \\
m_{2} s v_{2}+r_{1} v_{2}+v_{2} /\left(s c_{1}\right)=-F_{1}+r_{1} v_{1}+v_{1} /\left(s c_{1}\right)
\end{gathered}
$$

and for the Terfenol-D controlled low frequency section,

$$
\begin{gathered}
\left(m_{1}+m_{2}\right) s v_{1}+r_{2} v_{1}+v_{1} /\left(s c_{2}\right)=F_{2}+r_{2} v_{3}+v_{3} /\left(s c_{2}\right) \\
m_{3} s v_{3}+r_{2} v_{3}+v_{3} /\left(s c_{2}\right)=-F_{2}+r_{2} v_{1}+v_{1} /\left(s c_{2}\right) .
\end{gathered}
$$

In these equations, $m$ represents the mass, $r$ the damping, $c$ the mechanical compliance, $v$ the velocity, and $F$ the force, with $s$ being the Laplace derivative operator $j \omega$. When the system is excited in a broadband fashion, the two modes' resonant peaks overlap in the frequency domain. The model simulations of Figure 2 show that this creates a wide, relatively flat region in the head mass velocity response, a key criterion for propagating acoustic energy into a medium.

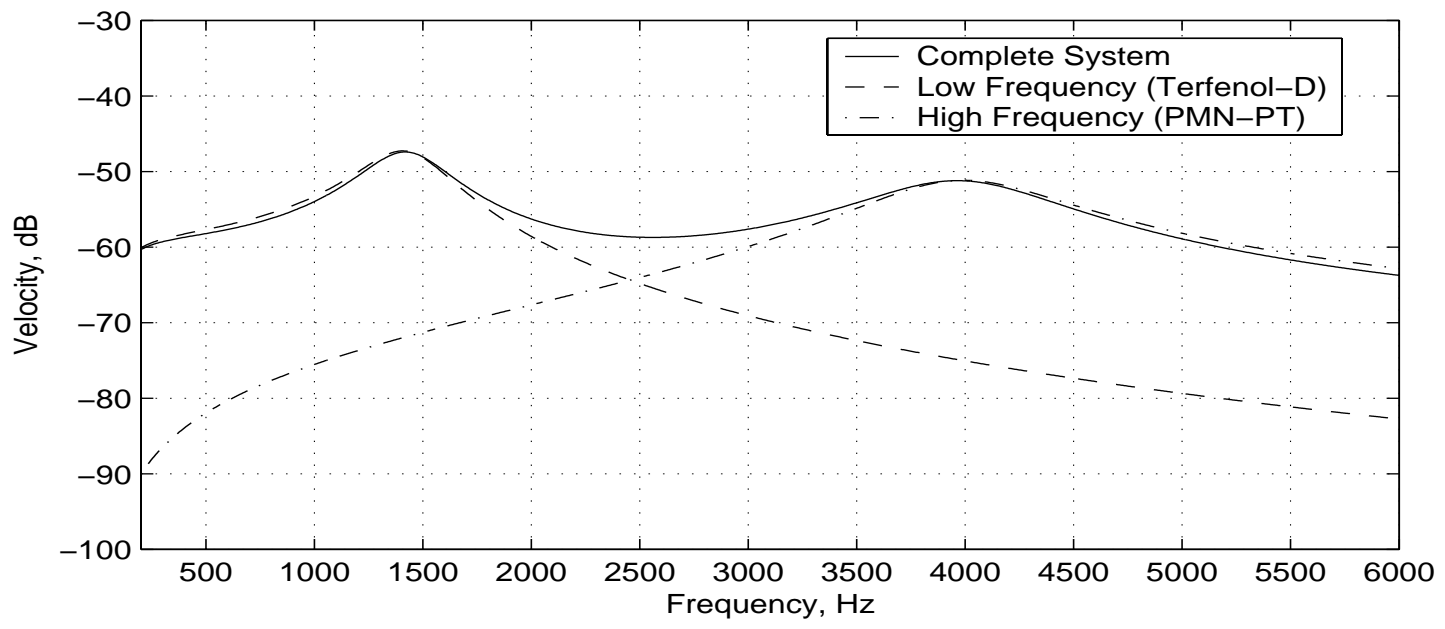

Figure 2: Head mass velocity response of the complete system and the individual sections calculated from equations (1) - (2).

\section{ELECTROACOUSTICS MODEL}

For the second modeling step, classical electroacoustic transduction theory ${ }^{8}$ is considered for purposes of coupling the vibratory model (1) - (2) with the electrical regime. For operating regimes in which linear models are accurate, the frequency domain relations that quantify the coupling between the electrical and mechanical components are

$$
\begin{aligned}
& V=Z_{e} I+T_{e m} v \\
& F=T_{m e} I+Z_{m} v,
\end{aligned}
$$

where $V$ is the voltage across the terminals, $I$ is the current through the transducer, $v$ is the velocity, $F$ the output force, $Z_{e}$ and $Z_{m}$ the blocked electrical and mechanical impedances, respectively, and $T_{e m}$ and $T_{m e}$ are transduction coefficients that describe the electromechanical coupling of the system. Assuming a load of impedance $Z_{L}$, equation ( $3 \mathrm{~b}$ ) can be rewritten in the form

$$
v=-T_{m e} I /\left(Z_{m}+Z_{L}\right)
$$

which upon substitution in equation (3a) gives the total electrical impedance,

$$
Z_{e e}=V / I=Z_{e}+\left(-T_{e m} T_{m e}\right) /\left(Z_{m}+Z_{L}\right)=Z_{e}+Z_{m o t}
$$


which represents the superposition of the blocked electrical impedance $Z_{e}$ and the coupled effects of the mechanical motion $Z_{\text {mot }}$.

By measuring $Z_{e e}$, the motional contributions can be used as a measure of performance for each active material. The total electrical admittance of the system, $Y_{e e}=1 / Z_{e e}$, can be analyzed along with the impedance to determine effective values for several parameters, such as the coupling, stiffness, and damping of each section. This analysis is done at the drive levels of interest so that the values obtained incorporate the natural variation of smart material properties with operating conditions ${ }^{9,10}$. This process will be detailed in Section 7.2.

\section{MATERIAL MODELS}

Linear electroacoustic relations (3) - (4) provide a framework which is sufficiently general to analyze a variety of electromechanical systems. For purposes of modeling hybrid transducers, however, additional physical details must be considered, especially concerning coupling effects within each active section and between the active sections. To this end, linearized constitutive relations for the active materials will be converted into a form comparable with (3), thus allowing for the blocked impedances and transduction coefficients to be expressed in terms of properties such as coupling coefficients, Young's moduli, magnetic permeabilities, and physical dimensions ${ }^{11}$. While the material behaviors are actually nonlinear and hysteretic, linearized models are sufficiently accurate for biased, low signal operation in which saturation and frequency-doubling effects are reduced. Additional assumptions in this analysis include uniform stress and strain, ideal electrical components, field uniformity in each section, and no hysteresis.

\subsection{Terfenol-D}

For biased, low signal operation, linear constitutive equations quantifying the direct and inverse magnetostrictive effects are

$$
\begin{gathered}
\varepsilon=\sigma / E_{y}{ }^{H}+q H \\
B=q \sigma+\mu^{\sigma} H,
\end{gathered}
$$

where $\varepsilon$ is the strain, $\varepsilon$ is the axial stress, $E_{y}{ }^{H}$ is the Young's Modulus at constant field strength, $q$ is the coupling coefficient, $\varepsilon^{\sigma}$ is the magnetic permeability at constant stress, $H$ is the applied magnetic field strength, and $B$ is the flux density. To convert these equations into a form comparable with equation (3), the following electromechanical relations are employed,

$$
\begin{gathered}
H=n I \\
\varepsilon=v /\left(j \omega L_{2}\right) \\
\sigma=F / A_{2} \\
V=R I+j \omega N A_{2} B
\end{gathered}
$$

where $n$ is the turns ratio of the magnetic drive coil $\left(n=N / L_{2}\right), L_{2}$ is the length of the coil and rod, $A_{2}$ is the rod crosssectional area (and internal area of the coil), and $R$ is the wire resistance. The total voltage drop $V$ is that of a DC resistance in series with an electrical inductance, which experimentally is found to be accurate. Substitution of (6) into (5) yields

$$
\begin{gathered}
V=\left(R+j \omega \mu^{\sigma}\left(1-k^{2}\right) n^{2} A_{2} L_{2}\right) I+N q k_{m}{ }^{H} v \\
F=-N q k_{m}{ }^{H} I+k_{m}{ }^{H} /(j \omega) v .
\end{gathered}
$$


Here, these equations are analogous to the electroacoustic relations (3). The term $k^{2}$ is the magnetomechanical coupling factor, defined as

$$
k^{2}=q^{2} E_{y}{ }^{H} / \mu^{\sigma},
$$

while $k_{m}{ }^{H}=1 / c 2$ is the mechanical stiffness defined in equation (2). From (7a), it is evident that the blocked electrical impedance is as expected for the wound wire coil, with the inductance now expressed in terms of material properties of the Terfenol-D rod. Finally, the two transduction coefficients, $T_{e m}$ and $T_{m e}$, take the form

$$
\begin{gathered}
T_{e m}=N q k_{m}{ }^{H} \\
T_{m e}=-N q k_{m}{ }^{H}=-T_{e m} .
\end{gathered}
$$

It is notable that they are opposite in sign while equal in magnitude. This antisymmetric relationship, according to $\mathrm{Hunt}^{8}$, is expected of all magnetostrictive transducers because of the orthogonallity of current and magnetic field.

\subsection{PMN-PT}

In this section, analogous relations are developed for the PMN-PT stack. For low field inputs $E$ about a bias field $E_{o}$, the constitutive behavior of individual layers can be approximated by the linear piezoelectric equations

$$
\begin{gathered}
\varepsilon=\sigma / E_{y}^{E}+d E \\
D=d \sigma+\varepsilon_{0} E
\end{gathered}
$$

where $E_{y}{ }^{E}$ is the Young's Modulus at constant electric field, $E$ is the electric field strength, $D$ is the electric displacement, $d$ is the linear coupling coefficient, and $\varepsilon_{o}$ is the permittivity. Relationships analogous to those derived for magnetostrictive materials have the form

$$
\begin{gathered}
E=V / t \\
\varepsilon=v /(j \omega t) \\
\sigma=F / A_{1} \\
D=I /\left(j \omega A_{1}\right),
\end{gathered}
$$

in which it is assumed that each layer has thickness $t$ and cross-sectional area $A_{l}$. Using these equations in (10) gives, for $N$ layers,

$$
\begin{gathered}
V=t /\left(j \omega A_{1} N\left(\varepsilon_{0}-E_{y}{ }^{E} d^{2}\right)\right) I-d E_{y}{ }^{E} /\left(j \omega N\left(\varepsilon_{0}-E_{y}{ }^{E} d^{2}\right)\right) v \\
F=-d E_{y}{ }^{E} /\left(j \omega N\left(\varepsilon_{0}-E_{y}{ }^{E} d^{2}\right)\right) I+\varepsilon_{0} A_{1} E_{y}{ }^{E} /\left(j \omega N\left(\varepsilon_{0}-E_{y}{ }^{E} d^{2}\right)\right) v
\end{gathered}
$$

which are in standard electroacoustic form given in (3). From these equations, it is inferred that the blocked electrical impedance of the stack, $Z_{e}$, is purely capacitive, and has the form of an ideal parallel plate capacitor with a dielectric medium. In these equations, it is also seen that the transduction coefficients are symmetric, that is they are identical in both magnitude and sign,

$$
\begin{gathered}
T_{e m}=-d E_{y}{ }^{E} /\left(j \omega N\left(\varepsilon_{0}-E_{y}{ }^{E} d^{2}\right)\right) \\
T_{m e}=-d E_{y}{ }^{E} /\left(j \omega N\left(\varepsilon_{0}-E_{y}{ }^{E} d^{2}\right)\right)=T_{e m .}
\end{gathered}
$$


This symmetry is consistent with the electrical properties of the system. It was mentioned that the velocities of the two sections were 90 degrees out of phase, and that this property is useful in extending bandwidth. This inherent phase shift can be proven by comparing the transduction coefficients (electrical to mechanical), $T_{e m}$, of each section,

$$
\angle\left(T_{e m}^{p m n} / T_{e m}{ }^{\text {terf }}\right)=\angle\left(-T_{1} /(j \omega) / T_{2}\right)=90^{\circ} .
$$

This transduction coefficient relates voltage to velocity in the electroacoustic framework. When the two sections are wired in parallel and receive the same applied voltage, the phase of the velocities will be determined by the $T_{e m}$ 's, and thus will always be 90 degrees apart, even when moving through resonance.

\section{COMBINED TRANSDUCER MODEL}

With relations for the mechanical, electroacoustic, and material regimes of each smart material section, it is necessary to combine all aspects of the model into an overall description of the transducer. The resultant equations from each model component need to be incorporated into a single governing relation for each smart material element. This expression, which includes all relevant electromechanical effects, is the total electrical impedance defined in equation (4). Once these expressions are formulated for each section, they need to be properly combined to fully describe the behavior of the total transducer.

From the mechanical model, equations (1) and (2) are used to derive $Z_{m}$, the blocked mechanical impedance, for each element. This is represented as force over velocity, where the velocity of interest is that of the head mass. Solving then for $F / v_{1}$ gives

$$
\begin{gathered}
Z_{m E}=F_{1} / v_{1}=\left(m_{1} m_{2} c_{1} s^{2}+\left(r_{1} c_{1} s+1\right)\left(m_{1}+m_{2}\right)\right) /\left(m_{2} c_{1} s\right) \\
Z_{m M}=F_{2} / v_{1}=\left(\left(m_{1}+m_{2}\right) m_{3} c_{2} s^{2}+\left(r_{2} c_{2} s+1\right)\left(m_{1}+m_{2}+m_{3}\right)\right) /\left(m_{3} c_{2} s\right),
\end{gathered}
$$

where all of the physical constants are defined from Figure 1, and subscript $E$ represents the PMN-PT and subscript $M$ the Terfenol-D. The material models (7) and (12) give the blocked electrical impedances,

$$
\begin{aligned}
& Z_{e E}=t /\left(j \omega A_{1} N\left(\varepsilon_{0}-E_{y}^{E} d^{2}\right)\right)=1 /\left(j \omega C_{\text {block }}\right) \\
& Z_{e M}=R+j \omega \mu^{\sigma}\left(1-k^{2}\right) n^{2} A_{2} L_{2}=R+j \omega L_{\text {block }}
\end{aligned}
$$

and the transduction coefficients defined in (9) and (13). The total electrical impedance, $Z_{e e}$, of each smart material section is then

$$
\begin{gathered}
Z_{e e E}=Z_{e E}+\left(-T_{e m E} T_{m e E}\right) /\left(Z_{m E}+Z_{L}\right) \\
Z_{e e M}=Z_{e M}+\left(-T_{e m M} T_{m e M}\right) /\left(Z_{m M}+Z_{L}\right) .
\end{gathered}
$$

The two are combined by recognizing that the sections are wired in parallel, and as such their total electrical impedances, with motional contributions, sum as expected. Thus, the complete hybrid system's overall electrical impedance, incorporating both elements' different electrical and mechanical effects, is

$$
Z_{e e}{ }^{\text {total }}=Z_{e e E} Z_{e e M} /\left(Z_{e e E}+Z_{e e M}\right)
$$

This can now be simulated and compared with actual test data, so long as reasonable values can be obtained for all of the material properties in the model equations. 


\section{TRANSDUCER DESIGN AND TESTING}

A broadband hybrid transducer was designed and constructed in the Smart Materials and Structures Lab at the Ohio State University. The magnetostrictive element consists of an ETREMA Terfenol-D rod ( Tb 0.73, Dy 0.27, Fe 1.95), $50.8 \mathrm{~mm}(2 \mathrm{in})$ in length and $6.35 \mathrm{~mm}(0.25 \mathrm{in})$ in diameter. The field is provided by a 1200 turn coil wound using 26AWG magnet wire which includes a single layer pick-up coil on the internal diameter. The coil is encased in a cylindrical Alnico V permanent magnet which provides a magnetic bias of $125 \mathrm{Oe}(10 \mathrm{kA} / \mathrm{m})$ and is axially slit to reduce eddy currents. Machined components of 1018 steel are used to complete a closed magnetic circuit path. The complete magnetic circuit is positioned between the $777 \mathrm{~g}$ tail mass and the $670 \mathrm{~g}$ center mass. Each mass is $76.2 \mathrm{~mm}(3 \mathrm{in})$ in diameter and made from non-magnetic stainless steel. Three bolts, each with Belleville washers under the head, are used to compress the magnetic components between the two masses, with the washers providing a means of applying a consistent compressive prestress to the Terfenol-D rod. The transducer's electrostrictive section employs an EDO Ceramic model EP200-62 EC-98 stack. This material is lead magnesium niobate - lead titanate in a 65\% - 35\% ratio, a composition for which the linear piezoelectric equations are accurate ${ }^{12}$. This ceramic was chosen for its stiffness value of approximately $120 \mathrm{e} 6 \mathrm{~N} / \mathrm{m}$ to create the two mechanical resonances desired when placed in series with the magnetostrictive section. The stack consists of 62 individual layers and has a total length of $35.2 \mathrm{~mm}$ (1.385 in) and a diameter of $16 \mathrm{~mm}(0.63 \mathrm{in}$.) The ceramic stack is located between the center mass and the $308 \mathrm{~g}$ head mass and three prestress bolts with Belleville washers are used to compress the stack and hold the structure together. The finished transducer has a length of $152.4 \mathrm{~mm}$ (6 in) and is shown in Figure 3 (a).

The experiments were conducted with the test transducer placed inside of a $76.2 \mathrm{~mm}$ ( $3 \mathrm{in}$ ) PVC tube, with a neoprene/cork ring around each mass acting as an isolator. As shown in Fig. 3 (b), for experimental testing the complete assembly is suspended to prevent exogenous dynamics from affecting the transducer data. Low frequency tests were conducted independently on the Terfenol-D and PMN-PT elements to determine the quasi-static linear coupling coefficients $q$ and $d$, and to find an adequate low signal range for driving the system. The strain vs. magnetic field 'butterfly' curve of the Terfenol-D rod was measured with a linear variable differential transducer (LVDT), and from it a magnetic bias and $\mathrm{AC}$ drive level were chosen to limit motion to the steepest linear region on the curve. All subsequent testing employed the magnetic bias so determined. Broadband tests were conducted using white noise excitation with an upper frequency span ranging from $5-20 \mathrm{kHz}$. The measured quantities in these tests include applied voltage and current, and acceleration at each mass. Tests were conducted with each individual section driven independently and with the two wired in parallel. Additional tests were conducted with a capacitor wired in parallel with the transducer for purposes of determining the effect of transducer capacitance on the electrical resonance frequency. Finally, the PVC tube was filled with duct seal to simulate the loading effect of water ${ }^{13}$. The hardware employed for data acquisition includes a Data Physics SignalStar Vector dynamic signal analyzer and accompanying Mobilyzer 4.0 software. Two Techron 7780 amplifiers connected in electrical series were used to drive the system with a voltage gain of 60, and a built-in current monitor was employed for recording the current flow into the transducer. A Lucas Schaevitz MHR-025 LVDT was used for low frequency measurements, while PCB U353B16 accelerometers were used for broadband tests.

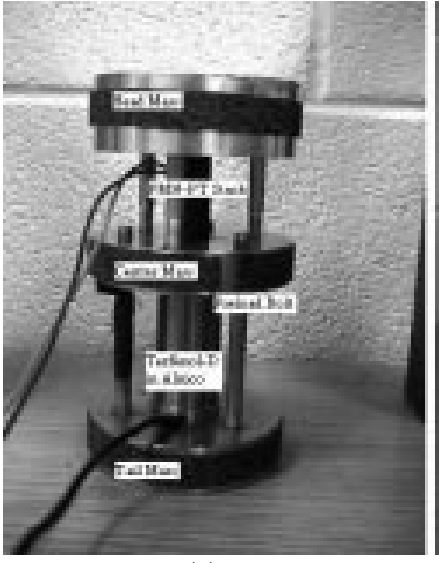

(a)

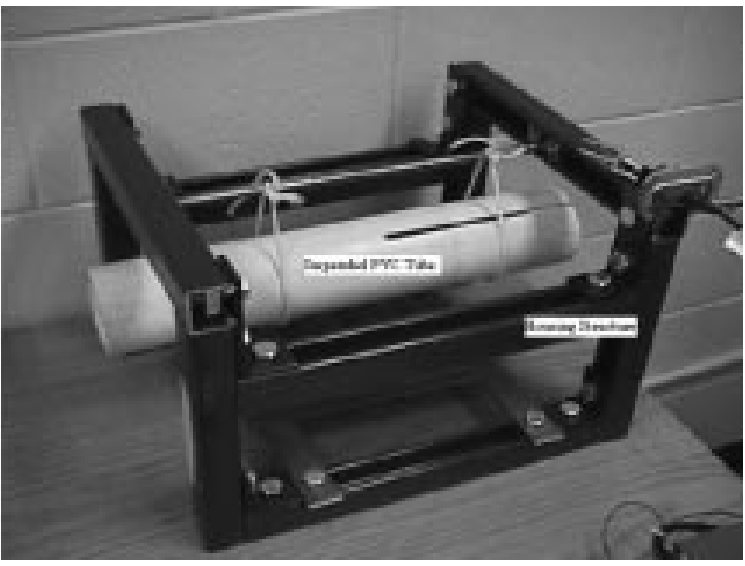

(b)

Figure 3: (a) Complete Terfenol-D/PMN-PT broadband transducer and (b) test bed structure. 


\section{RESULTS AND ANALYSIS}

\subsection{Low Frequency Terfenol-D Tests}

Quasi-static time domain tests were performed for purposes of determining the magnetic bias needed to obtain symmetric strains from the Terfenol-D rod. Figure 4 shows strain versus field and magnetic induction versus field curves. Each graph shows major as well as minor hysteresis loops, in which the latter represent the field drive condition employed in the subsequent dynamic tests. In the dynamic tests, the bias of $125 \mathrm{Oe}(10 \mathrm{kA} / \mathrm{m})$ provided by the Alnico magnet was increased to $220 \mathrm{Oe}(17.6 \mathrm{kA} / \mathrm{m})$ by applying $2.1 \mathrm{~V} \mathrm{DC}$ to the drive coil. The minor loops shown in Fig. 4 were obtained by applying +/- $2.1 \mathrm{~V}$ AC to the drive coil, which represents a total field of +/- $190 \mathrm{Oe}(15.2 \mathrm{kA} / \mathrm{m}) 0-\mathrm{pk}$.

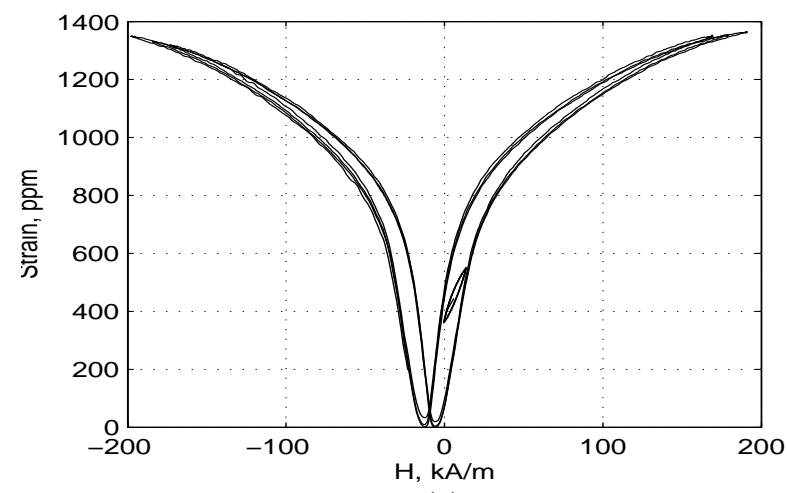

(a)

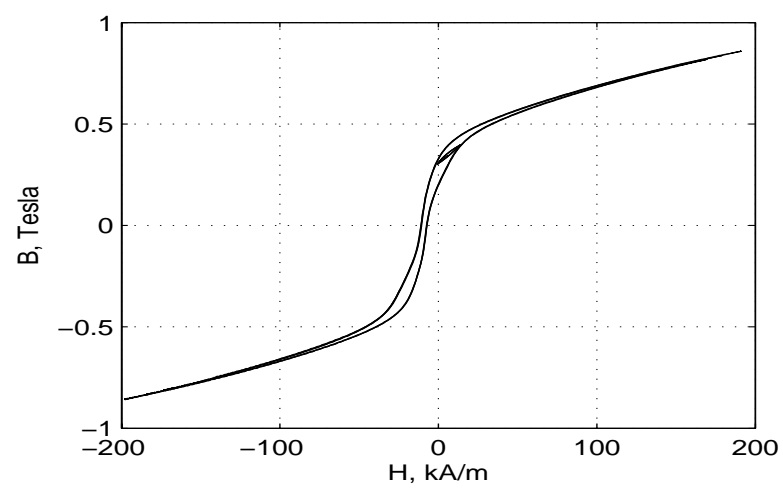

(b)

Figure 4: Major and minor Terfenol-D hysteresis loops (a) strain vs. magnetic field, and (b) magnetic induction vs. field.

\subsection{Electroacoustic Analysis}

Broadband electrical impedance and acceleration measurements were conducted through white noise voltage excitation in the $0-4.2 \mathrm{~V}$ range. Fig. 5 (a) shows a typical electrical impedance plot for the unloaded PMN-PT section, in which $Z_{e E}$ represents the blocked impedance and the deviation from this line represents the coupled motional effects described in Section 3. The corresponding Nyquist plots are shown in Figure 6, in which it is noted that frequency increases clockwise around each mobility circle.

In the impedance graph, the frequency directly opposite the crossover point is the resonance frequency and on the admittance graph, this point gives the anti-resonant frequency. Note that these frequencies do not directly correlate to the relative maximum and minimum magnitudes shown in Figure 5 (a) since those points do not contain phase information. The two points on the circles at 90 degrees from the main diameter are the half power points, a set each for the resonance and anti-resonance frequencies. A similar analysis was done for the Terfenol-D section as well as the complete transducer. It has been shown ${ }^{8}$ that the effective coupling of each section can be determined from the relations

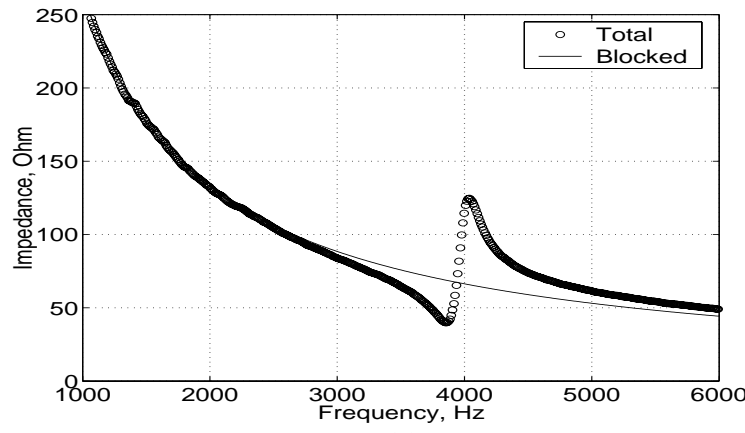

(a)

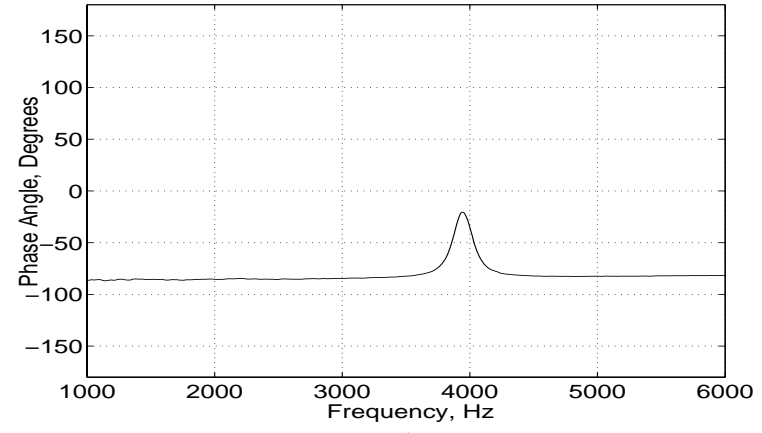

(b)

Figure 5: Total and blocked electrical impedance of the PMN-PT section, (a) magnitude and (b) phase. 


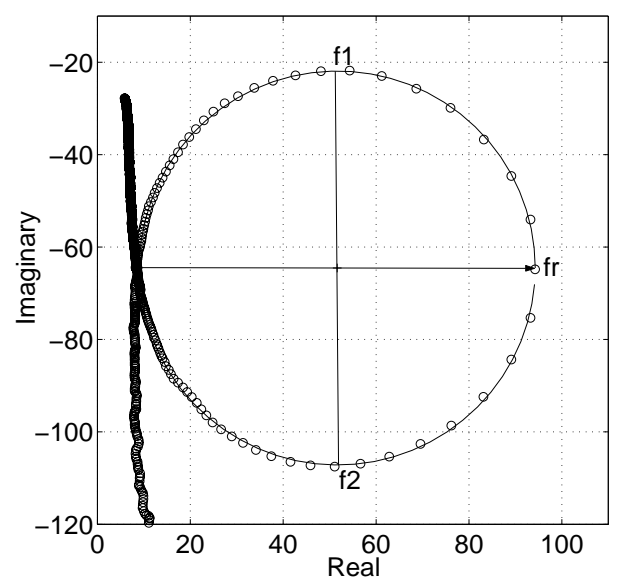

(a)

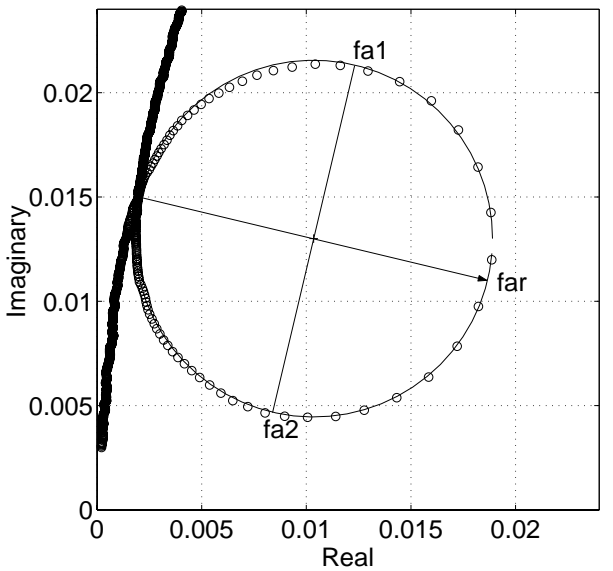

(b)

Figure 6: (a) Impedance and (b) admittance mobility loops showing resonance (fr), anti-resonance (far), and half power point $(\mathrm{f} 1, \mathrm{f} 2, \mathrm{fa} 1, \mathrm{fa} 2)$ frequencies.

$$
\begin{aligned}
& k_{\text {eff }}^{2}=1-\left(f_{a r} / f_{r}\right)^{2} \\
& {k_{\text {eff }}{ }^{2}}^{2}=1-\left(f_{r} / f_{a r}\right)^{2}
\end{aligned}
$$

respectively for the PMN-PT and Terfenol-D sections. Additionally, the resonant frequencies can be used in conjunction with the known masses to yield the stiffnesses of the active elements. From relations (14) for the mechanical impedances, resonance is determined by setting the imaginary component equal to zero, giving

$$
\begin{gathered}
k_{m}{ }^{E}=\left(2 \pi f_{r}\right)^{2} m_{1} m_{2} /\left(m_{1}+m_{2}\right) \\
k_{m}{ }^{H}=\left(2 \pi f_{r}\right)^{2}\left(m_{1}+m_{2}\right) m_{3} /\left(m_{1}+m_{2}+m_{3}\right),
\end{gathered}
$$

respectively for the PMN-PT and Terfenol-D. Assuming linear material behavior, the elastic moduli take the form

$$
\begin{aligned}
& E_{y}{ }^{E}=k_{m}{ }^{E} L_{1} / A_{1} \\
& E_{y}{ }^{H}=k_{m}{ }^{H} L_{2} / A_{2} .
\end{aligned}
$$

Relations (18) provide the coupling coefficients for the magnetostrictive and electrostrictive sections, but the effects of prestress and field leakage must be accounted for. Recognizing that each active element is arranged in mechanical parallel with the corresponding prestress stiffness, one obtains (after Hall ${ }^{11}$ )

$$
k^{2}=k_{e f f}^{2}\left(k_{m}+k_{m}^{p s}\right) /\left(k_{m} k_{L}+k_{e f f}^{2} k_{m}^{p s}\right)
$$

where $k_{m}$ is the section's stiffness obtained from (19), $k_{m}{ }^{p s}$ is the prestress stiffness, and $k_{L}$ represents flux leakage. This $k^{2}$ term is that expressed in equation (8) for the Terfenol-D, and thus is related directly to the material properties. Another property that can be found from this analysis is the mechanical quality factor $Q$ of each section,

$$
\begin{gathered}
Q_{r}=f_{r} /\left(f_{2}-f_{1}\right) \\
Q_{a r}=f_{a r} /\left(f_{a 2}-f_{a 1}\right),
\end{gathered}
$$


one from each set of frequencies, with $f_{2}$ and $f_{l}$ being the half power points associated with $f_{r}$, and $f_{a 2}$ and $f_{a l}$ those corresponding to $f_{a r}$. In this study, the average value of these $Q$ 's is used. From relations (14) for the mechanical impedances, the internal damping can be respectively estimated as

$$
\begin{gathered}
r_{1}=2 \pi f_{r} m_{1} m_{2} /\left(Q\left(m_{1}+m_{2}\right)\right) \\
r_{2}=2 \pi f_{r}\left(m_{1}+m_{2}\right) m_{3} /\left(Q\left(m_{1}+m_{2}+m_{3}\right)\right)
\end{gathered}
$$

for the PMN-PT and Terfenol-D sections. The complete procedure outlined in this section is repeated for each set of test data, with the calculated results used as nominal values in the model calculations.

\subsection{Broadband Model and Test Results}

The electrical impedance and head mass velocity graphs shown in Figure 7 were obtained by driving only the TerfenolD section while the PMN-PT section was left as an open circuit. The transducer was mechanically loaded by the duct seal material. The total modeled impedance underestimates the motional effects, primarily because of the errors associated with the estimated coupling coefficient. The larger actual impedance output, combined with knowledge that equations (18) become worse approximations under heavy resistive loading ${ }^{11}$, imply that the resultant estimated coupling coefficient is less than the actual. The effect of the low estimate can be seen in many of the following graphs as well. For example, the velocity response measurements suggest that the Terfenol-D section excites some motion of the PMNPT mode, where the model does not provide such response due to lack of complete coupling.

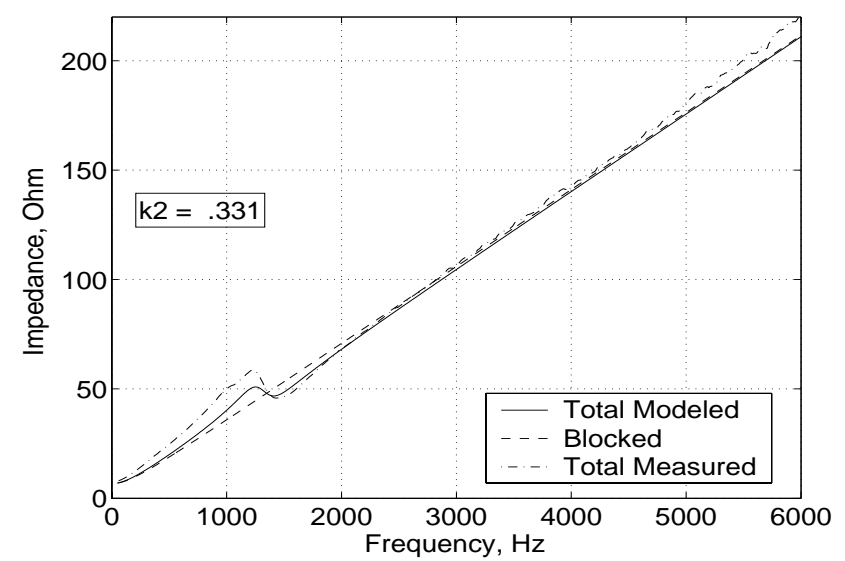

(a)

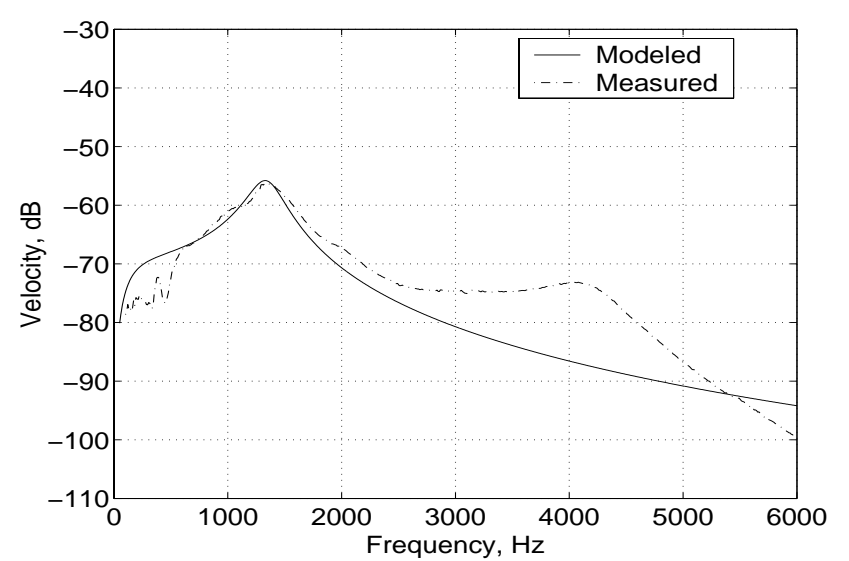

(b)

Figure 7: System response with only Terfenol-D excitation, (a) electrical impedance magnitudes, and (b) head mass velocity response.

Figure 8 displays the results of driving just the PMN-PT section, with the magnetic drive solenoid left open. The electrical impedance magnitude shows that the coupled motional effects are damped out compared with Figure 5 (a), owing to the external duct seal loading. This increased damping is even more evident in the velocity graph given the flat shape of the resonance peak.

When the two sections are driven simultaneously, the head mass velocity calculations match fairly well with the experimental measurements. The impedance curve for this test reveals that the natural electrical resonance of the system is located at a higher frequency than both of the mechanical peaks, such that the electrical resonance is not a factor over the mechanical bandwidth of interest. To see the effect of changing this electrical resonance, an external capacitor is wired in parallel to shift the electrical resonance to in between the two mechanical resonance frequencies. Figure 9 shows that in terms of the electrical impedance, the active sections are being driven like they were individually, while the head mass velocity response is the combination of the two individual responses, extending the bandwidth to the desired range. It is emphasized that the velocity response appears unaffected by the shift in electrical resonance, thus indicating that employing a different electrostrictive or piezoelectric element would not fundamentally change the transducer's bandwidth. 


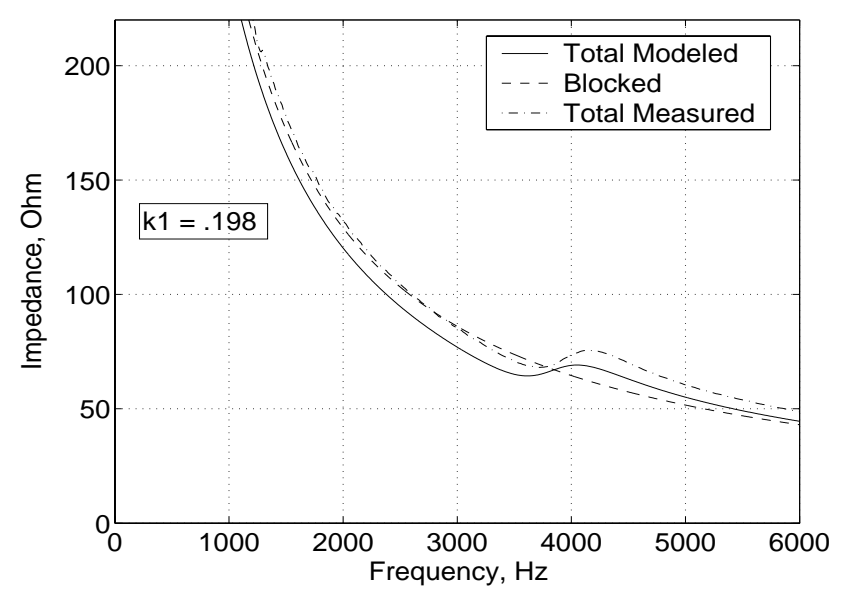

(a)

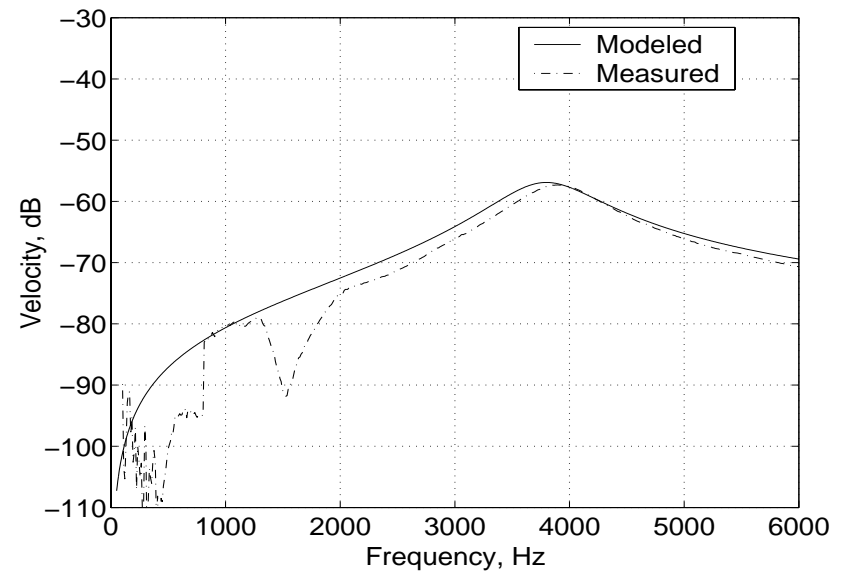

(b)

Figure 8: System response with only PMN-PT excitation, (a) electrical impedance magnitudes, and (b) head mass velocity response.

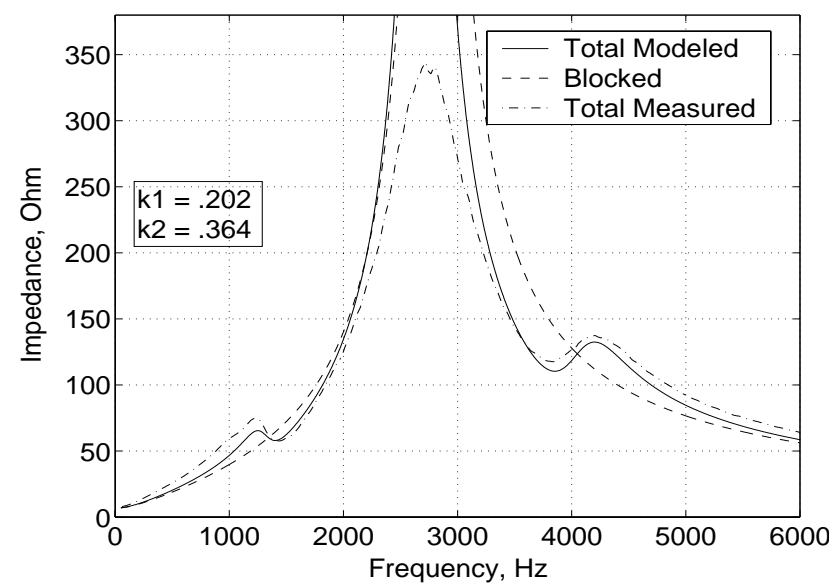

(a)

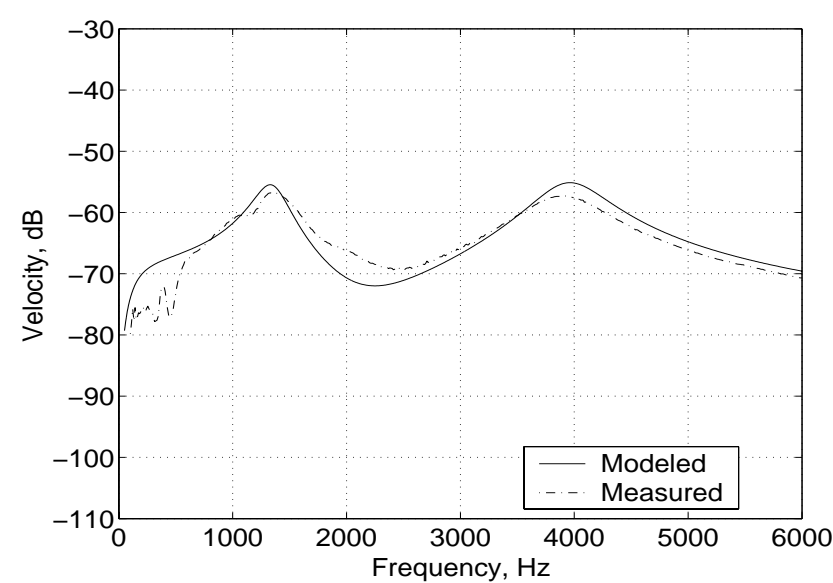

(b)

Figure 9: System response with both sections and added capacitor, (a) modeled and measured electrical impedance magnitudes, and (b) head mass velocity response.

Finally, the same setup is tested when the transducer is removed from the mechanical load impedance. In this configuration, the motional contributions to the electrical impedance are much larger, implying better coupling, but the velocity peaks become sharper as well, thus reducing the transducer's frequency bandwidth. It is noted that the model accurately predicts the system damping in this unloaded case, where it slightly underestimates the damping level in the loaded configuration illustrated in Figure 9 (b). This may be a result of the fact that relation (20) is best suited for lightly damped systems ${ }^{14}$.

\section{CONCLUSIONS}

A linear model was developed that completely describes the electrical and mechanical domains of a broadband magnetostrictive/electrostrictive transducer, and a test device was constructed using the model as a design tool. The transducer demonstrated the extension of the low end of the frequency spectrum to below $1 \mathrm{kHz}$. Experimental data was employed both for model parameter identification and validation. Both simulation and test data agree on the effects of altering the electrical resonance and the acoustic load. Notably, it was found that the velocity response is unaffected by electrical resonance shifts, which has design implications as it allows the use of different electrostrictive or piezoelectric elements without fundamentally diminishing the transducer's ability to provide broadband response. Future work will involve the enhancement of the system model by accounting for various nonlinearities in the dynamics and coupling of the constituent active materials. 


\section{ACKNOWLEDGEMENTS}

Support for P.R.D. and M.J.D. comes in part from the Ohio State University through startup funds. The research of R.C.S. is supported in part by the NSF grant CMS-0099764 and by the Air Force Office of Scientific Research under the grant AFOSR-F49620-01-1-0107. The authors wish to acknowledge Julie Slaughter of ETREMA Products Inc. for supplying a Terfenol-D rod on an in-kind basis, Stephen Butler for helpful suggestions, and David Moody for providing assistance during the initial stages of this investigation.

\section{REFERENCES}

1. H. Janocha, and B. Clephas, "Hybrid Actuator with Piezoelectric and Magnetostrictive Material," ACTUATOR 96, Proc. $5^{\text {th }}$ International Conference on New Actuators, pp. 304-307, 1996.

2. S.C. Butler, and F.A. Tito, "A Broadband Hybrid Magnetostrictive/Piezoelectric Transducer Array," OCEANS 2000 MTS/IEEE, Vol. 3, pp. 1469-1475, 2000.

3. M.J. Dapino, R.C. Smith, and A.B. Flatau, "Structural Magnetic Strain Model for Magnetostrictive Transducers," IEEE Trans. Magn., Vol. 36, pp. 545-556, 2000.

4. M.J. Dapino, R.C. Smith, L.E. Faidley, and A.B. Flatau, "Coupled Structural-Magnetic Strain and Stress Model for Magnetostrictive Transducers," Journal of Intelligent Material Systems and Structures, Vol. 11, pp. 135-152, 2000.

5. R.C. Smith, M.J. Dapino, and S. Seelecke, "Free energy model for hysteresis in magnetostrictive transducers," Journal of Applied Physics, Vol. 93, pp. 458-466, 2003.

6. C.L. Hom, and N. Shankar, "A Fully Coupled Constitutive Model for Electrostrictive Ceramic Materials," Journal of Intelligent Material Systems and Structures, Vol. 5, pp. 795-801, 1994.

7. J.C. Piquette, and S.E. Forsythe, "Generalized material model for lead magnesium niobate (PMN) and an associated electromechanical equivalent circuit," Journal of the Acoustical Society of America, Vol. 104, pp. 2763-2772, 1998.

8. F.V. Hunt, Electroacoustics: The Analysis of Transduction, and its Historical Background, American Institute of Physics for the Acoustical Society of America, 1982.

9. M.J. Dapino, F.T. Calkins, and A.B. Flatau, "On identification and analysis of fundamental issues in Terfenol-D transducer modeling," Proc. SPIE, Vol. 3329, pp. 185-197, 1998.

10. W. Ren, A.J. Masys, G. Yang, and B.K. Mukherjee, "The Variation of Piezoelectric and Electrostrictive Strain as a Function of Frequency and Applied Electric Field using Interferometric Technique," Proc. IEEE Int. Symposium on Applications of Ferroelectrics, Vol. 1, pp. 85-88, 2000.

11. D. Hall, "Dynamics and vibrations of magnetostrictive transducers," Ph.D. dissertation, Iowa State Univ., 1994.

12. O. Noblanc, C. Deljurie, P. Gaucher, and G. Calvarin, "Propriétés diélectriques et piézoélectriques de céramiques de PMN-PT autour de la composition morphotropique en relation avec la structure crystalline," Silicates Industriels, Vol. 11-12, pp. 227-231, 1993.

13. J.L. Butler, S.C. Butler, and A.E. Clark, "Unidirectional Magnetostrictive/Piezoelectric Hybrid Transducer," Journal of the Acoustical Society of America, Vol. 88, July 1990.

14. L. Meirovitch, Fundamentals of Vibrations, McGraw Hill, New York, 2001. 